\title{
Experimental high-intensity three-photon entangled source
}

\author{
Huai-Xin Lu, ${ }^{1}$ Jun Zhang, ${ }^{2}$, $⿴ 囗$ Xiao-Qin Wang, ${ }^{1}$ Ying-De Li, ${ }^{1}$ and Chun-Yan Wang ${ }^{1}$ \\ ${ }^{1}$ Department of Physics and Electronic Science, Weifang University, Weifang, Shandong 261061, China \\ ${ }^{2}$ Hefei National Laboratory for Physical Sciences at Microscale and Department of Modern Physics, \\ University of Science and Technology of China, Hefei, Anhui 230026, China
}

(Dated: December 3, 2018)

\begin{abstract}
We experimentally realize a high-intensity three-photon Greenberger-Horne-Zeilinger (GHZ) entanglement source directly following the proposal by Rarity and Tapster [J. G. Rarity and P. R. Tapster, Phys. Rev. A 59, R35 (1999)]. The threefold coincidence rate can be more than $200 \mathrm{~Hz}$ with a fidelity of 0.811 , and the intensity can be further improved with moderate fidelity degradation. The GHZ entanglement is characterized by testing the Bell-Mermin inequality and using an entanglement witness operator. To optimize the polarization-entangled source, we theoretically analyze the relationship between the mean photon number of the single-photon source and the probability of parametric down-conversion.

PACS numbers: 42.50.Dv, 03.65.Ud, 03.67.Mn, 03.67.Hk
\end{abstract}

\section{INTRODUCTION}

Recently optical quantum-information processing has been developed rapidly both theoretically and experimentally, where photonic entanglement, especially multiphoton entanglement [1] plays a crucial role not only in the fundamental tests of quantum nonlocality [2], but also in optical quantum computation [3, 4] and quantum teleportation [5], multiparty communication [18], quantum key distribution [6]. As we know, two-photon maximally entangled state, or Bell state, can violate the Bell inequality [2] to show the inconsistency between quantum mechanics (QM) and local reality (LR) theory, and so far there are numerous experiments to verify the validity of quantum mechanics. However, entanglement of more than two photons, i.e., multiphoton maximally entangled state, or GHZ state [7, 8] can demonstrate the conflicts deterministically and nonstatistically, which are stronger and more straightforward than two-photon entanglement.

Several groups have experimentally realized the creation and manipulation of multiphoton entanglement, from three-photon [9], four-photon [10], five-photon [11] to six-photon 12] entanglement. However, all of the reported multiphoton entangled sources so far are hard to be applied to long-distance quantum communication due to the limited intensity of the sources.

In this Letter, we report a high intensity of threephoton polarization-entangled source following the proposal by Rarity and Tapster [13]. It is brighter than the intensities of publicly reported three-photon entanglement in all the previous experiments so far. Although in our experiment we use the same techniques as the previous experiments of multiphoton entanglement, here we obtain brighter three-photon entanglement with bet-

\footnotetext{
* Present address: Group of Applied Physics, University of Geneva,
} CH-1211 Geneva 4, Switzerland ter quality through careful improvements and optimizations. Our results suggest that the three-photon entangled source can be used for multiparty long-distance quantum communication.

\section{EXPERIMENT}

The experimental setup is shown in Fig. 1. The cw $532 \mathrm{~nm}$ all solid-state green laser (Millennia Pro 10s, Newport Co.) pumps the mode-locked Ti:sapphire laser (Tsunami, Newport Co.) and generates femtosecond pulses at the central wavelength of $780 \mathrm{~nm}$, with a repetition rate of $80 \mathrm{MHz}$ and a pulse width of $\sim 100 \mathrm{fs}$. In the experiment, the power of pump laser is $8.5 \mathrm{~W}$ and the output power of pulsed red laser is $\sim 1.4 \mathrm{~W}$. Then the pulsed laser passes through the $\mathrm{LiB}_{3} \mathrm{O}_{5}$ (LBO) crystal to produce ultraviolet (uv) laser at the central wavelength of $390 \mathrm{~nm}$ after the up-conversion process. A lens is inserted before the LBO crystal to form a small focused beam on the crystal and improve the up-conversion efficiency. Since the output beam from the laser is elliptical, we use the combination of two cylindrical lens to reshape the beam to be circular. Due to the limited efficiency of up-conversion, the beam after the LBO crystal is mixed by the uv and unconverted red laser. In order to effectively separate them, five dichroic mirrors (DM), which can reflect uv laser while transmitting red laser, are used. The power of the transmitted unconverted $780 \mathrm{~nm}$ laser from the first DM is more than $500 \mathrm{~mW}$. It is strongly attenuated by a series of attenuators to simulate a single-photon source (SPS). The uv laser of $\sim 400 \mathrm{~mW}$ traverses a 2 -mm-thickness $\beta$ - $\mathrm{BaB}_{2} \mathrm{O}_{4}$ (BBO) crystal to generate polarization-entangled photon pairs owing to the process of spontaneous parametric down-conversion 14]. Through optimizing the collection efficiency, the observed count rate of photon pairs is more than $18 \mathrm{kHz}$, with a compensator composed of a half-wave-plate and a 1-mm-thickness BBO crystal, and a $3 \mathrm{~nm}$ bandwidth interferometer filter (IF) in each 


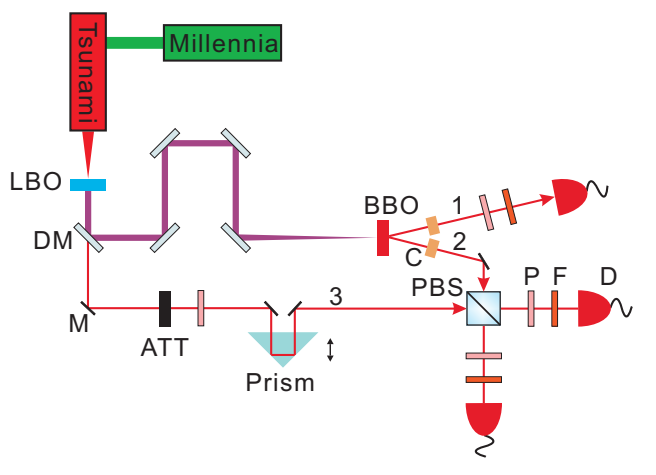

FIG. 1: (Color online) Experimental setup. ATT, attenuator; M, mirror; C, compensator; P, polarizer; F, filter; D, detector.

side. The prepared two-photon polarization-entangled state can be written as

$$
|\Psi\rangle_{12}=1 / \sqrt{2}\left(|H\rangle_{1}|V\rangle_{2}+|V\rangle_{1}|H\rangle_{2}\right),
$$

where $|H\rangle,|V\rangle$ present horizontal and vertical polarization of photons respectively. During the experiment, the visibility of two-photon entangled state is $95 \%$ in the $\mathrm{H} / \mathrm{V}$ basis while $94 \%$ in the $+/$ - basis, with $|+\rangle=$ $1 / \sqrt{2}(|H\rangle+|V\rangle)$ and $|-\rangle=1 / \sqrt{2}(|H\rangle-|V\rangle)$.

The polarization of a single photon in path 3 is fixed at $|+\rangle$ and sent to a polarization beam splitter (PBS), which transmits horizontal polarization while reflecting vertical polarization. After carefully adjusting the position of the prism controlled by a precise stepper motor, photon 2 and photon 3 can be completely overlapped on the PBS both spatially and temporally. When the polarization of the two photons is identical, we can prepare the three-photon GHZ entanglement as follows:

$$
\left.\left.|\Psi\rangle_{123}=1 / \sqrt{2}\left(|H\rangle_{1}|V\rangle_{2} V\right\rangle_{3}+|V\rangle_{1}|H\rangle_{2} H\right\rangle_{3}\right),
$$

since we can slightly tilt the BBO crystal in one of the compensators to preserve that the phase of the state is zero.

The eight coincidence components are shown in Fig. 2(a), where the undesired components are much less than the desired coincidences of $\mathrm{H}_{1} \mathrm{~V}_{2} \mathrm{~V}_{3}$ and $\mathrm{V}_{1} \mathrm{H}_{2} \mathrm{H}_{3}$. In order to confirm the coherent superposition of the three-photon GHZ state and observe the two-photon interference effect [15], we first move the position of the prism and then perform the coincident measurement on the three photons in the +/- basis, which is plotted in Fig. 2(b).

We have emphasized that the GHZ state can explicitly show the conflicts between QM and LR. Consider the following four joint measurements on the three-photon GHZ state:

$$
\sigma_{x 1} \sigma_{x 2} \sigma_{x 3}, \sigma_{x 1} \sigma_{y 2} \sigma_{y 3}, \sigma_{y 1} \sigma_{x 2} \sigma_{y 3}, \sigma_{y 1} \sigma_{y 2} \sigma_{x 3},
$$

where $\sigma_{x}, \sigma_{y}$ are the Pauli operators, which can be experimentally measured in the $+/-$ and $\mathrm{R} / \mathrm{L}$ basis, respectively, with $|R\rangle=1 / \sqrt{2}(|H\rangle+i|V\rangle)$ and $|L\rangle=$
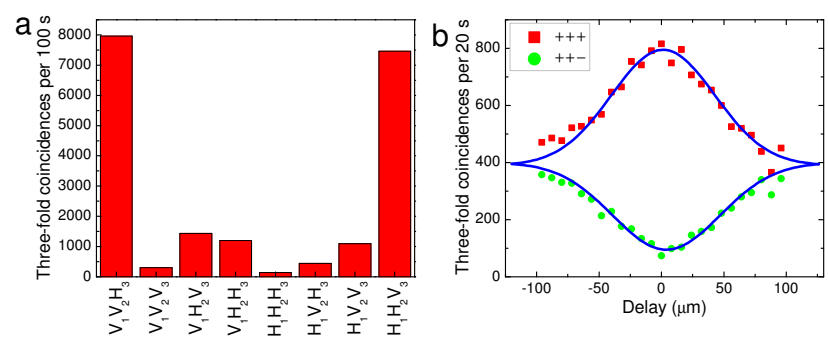

FIG. 2: (Color online) (a) Eight coincidence components in the $\mathrm{H} / \mathrm{V}$ basis. (b) Experimental observation of two-photon Hong-Ou-Mandel interference. Points and solid lines present experimental values and theoretical fits. Threefold coincidences in the +/- basis are measured as a function of the delay position of the prism. At the zero delay, the interference visibility is more than 0.79 , which is sufficient to imply the coherent superposition of three photons.

$1 / \sqrt{2}(|H\rangle-i|V\rangle)$. Therefore, we can easily measure the above four joint operators, e.g., $\sigma_{x 1} \sigma_{y 2} \sigma_{y 3}$ implies that photon 1 is measured in the $+/$ - basis while photon 2 and photon 3 are both measured in the R/L basis simultaneously. Then we can experimentally test the Bell-Mermin inequality [16] by

$$
M=\sigma_{x 1} \sigma_{x 2} \sigma_{x 3}+\sigma_{y 1} \sigma_{x 2} \sigma_{y 3}+\sigma_{y 1} \sigma_{y 2} \sigma_{x 3}-\sigma_{x 1} \sigma_{y 2} \sigma_{y 3} .
$$

QM and LR will predict completely different results for this inequality with

$$
\left|<M>_{L R}\right| \leq 2, \quad\left|<M>_{Q M}\right| \leq 4 .
$$

That is to say, the expectation value of $M$ predicted by any LR theory cannot be larger than 2 . We verify this by performing polarization measurements on the state. For example, to measure the expectation value of the $\sigma_{x 1} \sigma_{y 2} \sigma_{y 3}$ operator, eight sorts of polarization settings $(+R R,+R L,+L R,+L L,-R R,-R L,-L R$, and $-L L)$ must be performed. The result is shown in Fig. 3 and the experimental value of $M$ is $M_{E}=3.4113 \pm 0.0054$, which shows a violation of LR with more than 260 standard deviations. This also indicates that the prepared state in the experiment is a genuine GHZ state.

In fact, except for using the Bell-Mermin inequality to validate the GHZ entanglement, there are some other approaches to characterize the multipartite entanglement such as quantum state tomography, entanglement witness. We can quantify the quality of the produced GHZ state by evaluating the fidelity as defined by

$$
\mathcal{F}=\operatorname{Tr}\left(\rho_{\text {exp }}\left|\Psi_{123}\right\rangle\left\langle\Psi_{123}\right|\right),
$$

where $\rho_{\text {exp }}$ is the density matrix of the produced state. The fidelity can be determined by the local measurements on individual qubits. The experimental expectation value of fidelity is $\mathcal{F}_{E}=0.811 \pm 0.002$.

Furthermore, we can use the following entanglement witness operator [17] to detect the GHZ entanglement:

$$
\mathcal{W}=\frac{1}{2} \mathbb{1}-|\Psi\rangle_{123}\langle\Psi|,
$$




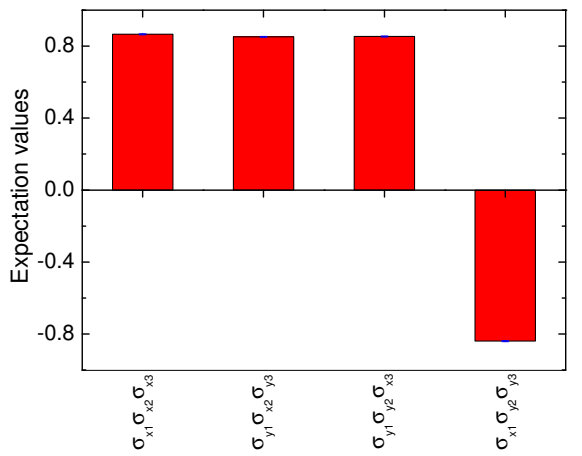

FIG. 3: (Color online) Experimental results of testing the Bell-Mermin inequality.

where $\mathbb{1}$ is an identity operator and $|\Psi\rangle_{123}\langle\Psi|$ is a projection operator to the GHZ state. If the expectation value of this witness operator is negative, it implies the existence of genuine GHZ entanglement. The expectation value of $\mathcal{W}$ is

$$
\mathcal{W}_{E}=\frac{1}{2}-\mathcal{F}_{E}
$$

whose experimental value is $\mathcal{W}_{E}=-0.311 \pm 0.002$, which is negative with about 155 standard deviations. This clearly proves the prepared state is truly entangled.

\section{EXPERIMENTAL ANALYSIS}

The single-photon source in path 3 is not a strictly true single-photon emitter, but a weak coherent laser. The mean photon number $\mu$ of the SPS will dominate the intensity of the three-photon entangled source. Higher $\mu$ will increase the intensity while decrease the visibility of the entangled source. Thus optimization of the $\mu$ value is necessary. Here we give a detailed analysis, see Fig. [4 The total count rates emitted from the SPS and EinsteinPodolsky-Rosen (EPR) pairs are

$$
N_{S}=f \mu, \quad N_{E}=f P_{E},
$$

where $f$ is the repetition frequency of the optical pulses, i.e., $80 \mathrm{MHz}$, and $P_{E}$ is the probability of downconversion per pulse in the nonlinear crystal. For simplicity, we assume that the coupling efficiencies of the three paths are equal, say, $\eta_{C}$, and the detection efficiencies of the three detectors are also equal, say, $\eta_{D}$, and the term of $e^{-\mu}$ is omitted. Then we can estimate the detected count rates of $H_{3^{\prime}}, V_{3^{\prime}}, H_{3^{\prime}} V_{3^{\prime}}$ from the SPS, and $H_{1}$, $V_{2^{\prime}}, V_{1}, H_{2^{\prime}}, H_{1} V_{2^{\prime}}, V_{1} H_{2^{\prime}}$ from the EPR, respectively, given by

$$
\begin{aligned}
& N_{S\left(H_{3^{\prime}}\right)}=N_{S\left(V_{3^{\prime}}\right)}=\frac{1}{2} f \mu \eta_{C} \eta_{D}, \\
& N_{S\left(H_{3^{\prime}} V_{3^{\prime}}\right)}=N_{S\left(V_{3^{\prime}} H_{3^{\prime}}\right)}=\frac{1}{2} f \mu^{2} \eta_{C}^{2} \eta_{D}^{2} \times \frac{1}{4}=\frac{1}{8} f \mu^{2} \eta_{C}^{2} \eta_{D}^{2}, \\
& N_{E\left(H_{1}\right)}=N_{E\left(V_{2^{\prime}}\right)}=N_{E\left(V_{1}\right)}=N_{E\left(H_{2^{\prime}}\right)}=\frac{1}{2} f P_{E} \eta_{C} \eta_{D},
\end{aligned}
$$

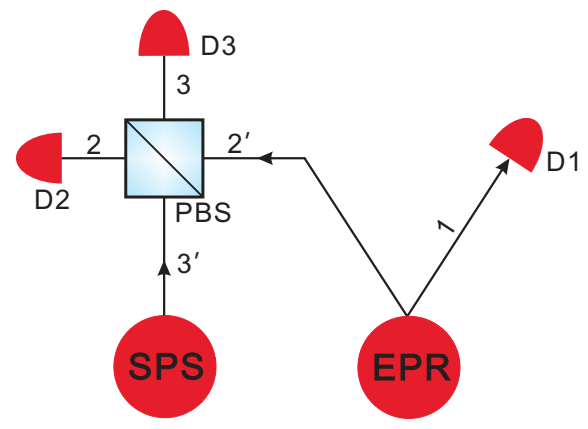

FIG. 4: (Color online) Simplified scheme for the three-photon entangled source. SPS and EPR present the single-photon source and two-photon entangled pairs respectively.

$$
N_{E\left(H_{1} V_{2^{\prime}}\right)}=N_{E\left(V_{1} H_{2^{\prime}}\right)}=\frac{1}{2} f P_{E} \eta_{C}^{2} \eta_{D}^{2},
$$

where we assume that the PBS is ideal and the insertion losses of other optical components are negligible. In the count rate of $N_{S\left(H_{3^{\prime}} V_{3^{\prime}}\right)}$, the factor of $\frac{1}{2} \mu^{2}$ is due to the consideration of Poisson distribution (the generation probability of two photons from the SPS is $\left.e^{-\mu} \mu^{2} / 2\right)$, and the four sorts of distributions of the two photons from the SPS $\left(H_{3^{\prime}} V_{3^{\prime}}, V_{3^{\prime}} H_{3^{\prime}}, H_{3^{\prime}} H_{3^{\prime}}, V_{3^{\prime}} V_{3^{\prime}}\right)$ result in the factor of $\frac{1}{4}$ in $N_{S\left(H_{3^{\prime}} V_{3^{\prime}}\right)}$.

When photon $2^{\prime}$ and photon $3^{\prime}$ are both $H$ or $V$ polarization, and the two photons are completely overlapped on the PBS, the three-photon GHZ entangled state can be created and its count rate is

$$
\begin{aligned}
N_{H_{1} V_{2} V_{3}} & =N_{V_{1} H_{2} H_{3}}=\frac{N_{E\left(H_{1} V_{2^{\prime}}\right)} N_{S\left(H_{3^{\prime}}\right)}}{f} \\
& =\frac{1}{4} f P_{E} \mu \eta_{C}^{3} \eta_{D}^{3} .
\end{aligned}
$$

Except the above two components, the other six sorts of the undesired components will also contribute to the threefold coincidences. When $H_{3^{\prime}}$ photons are transmitted while $V_{3^{\prime}}$ photons are reflected by the PBS, it will produce the following coincidence:

$$
\begin{aligned}
N_{H_{1} V_{2} H_{3}} & =N_{V_{1} V_{2} H_{3}}=\frac{N_{E\left(H_{1}\right)}\left[N_{S\left(H_{3^{\prime}} V_{3^{\prime}}\right)}+N_{S\left(V_{3^{\prime}} H_{3^{\prime}}\right)}\right]}{f} \\
& =\frac{1}{8} f P_{E} \mu^{2} \eta_{C}^{3} \eta_{D}^{3} .
\end{aligned}
$$

Similarly, when $H_{2^{\prime}}$ photons and $V_{2^{\prime}}$ photons pass the PBS it can cause the other two components

$$
\begin{aligned}
N_{H_{1} H_{2} V_{3}} & =N_{V_{1} H_{2} V_{3}}=\frac{N_{E\left(H_{1} V_{2^{\prime}}\right)} N_{E\left(H_{2^{\prime}}\right)}}{f} \\
& =\frac{1}{4} f P_{E}^{2} \eta_{C}^{3} \eta_{D}^{3} .
\end{aligned}
$$

The other undesired components of $H_{1} H_{2^{\prime}}, V_{1} V_{2^{\prime}}$ can also cause the following coincidence

$$
\begin{aligned}
N_{H_{1} H_{2} H_{3}} & =N_{V_{1} V_{2} V_{3}}=\frac{N_{E\left(H_{1}\right)} N_{E\left(H_{2^{\prime}}\right)} N_{S\left(H_{3^{\prime}}\right)}}{f^{2}} \\
& =\frac{1}{8} f P_{E}^{2} \mu \eta_{C}^{3} \eta_{D}^{3} .
\end{aligned}
$$


We can define a parameter $\mathcal{R}$ as the ratio of the desired coincidences to the undesired coincidences

$$
\begin{aligned}
\mathcal{R} & =\frac{2 N_{H_{1} V_{2} V_{3}}}{2\left(N_{H_{1} V_{2} H_{3}}+N_{H_{1} H_{2} V_{3}}+N_{H_{1} H_{2} H_{3}}\right)} \\
& =\frac{2 \mu}{\mu^{2}+2 P_{E}+P_{E} \mu} .
\end{aligned}
$$

Obviously, when $\mathcal{R}$ is larger the visibility of the GHZ state will be better. When $\mu \simeq \sqrt{2 P_{E}}, \mathcal{R}$ will reach the maximum value and the visibility of the three-photon entangled state is the best. We experimentally verify this theoretical result through controlling the $\mu$ value of the SPS. We find out when $\mu_{e}=\mu \eta_{C} \eta_{D} \sim 0.014$ the visibility of the entangled state can reach a quite good value. While during the experiment, we estimate $P_{E} \sim 0.022$ and $\eta_{C} \eta_{D}$ in path 3 is $\sim 0.1$, therefore the theoretical value of $\mu_{e}$ is 0.021 . The variance between the theoretical and experimental values is mainly due to that the calibration for the coupling efficiency in path 3 is not precise.

If $\mu$ is increased over the optimal value, the intensity of the three-photon entangled source is also increased with the cost of the decrease of the visibility. We observe the intensity to be more than $450 \mathrm{~Hz}$ with a few percentages of visibility degradation, which is larger than the intensity in the first three-photon experiment 9] with more than four orders of magnitude. On the other hand, in the experiment we have tried to improve the pump of the laser system up to the maximum power, $\sim 10.5 \mathrm{~W}$. Through careful optimization, the uv power can be increased to $\sim 500 \mathrm{~mW}$ and the intensity of two-photon entangled pairs is close to $30 \mathrm{kHz}$, which also implies that we can boost the intensity of the three-photon entangled source up to $\sim 1 \mathrm{kHz}$ with a moderate visibility. However, the laser system cannot be stable with this pump power for long-term experiment. This high intensity of three-photon entangled source is very valuable for many multiparty quantum communication protocols [18] and other applications in quantum-information processing [? ].

\section{CONCLUSION}

In summary, we have experimentally implemented a bright three-photon polarization-entangled source and tested the Bell-Mermin inequality to show the conflicts between quantum mechanics and local realism. We also introduce an entanglement witness operator to characterize three-photon GHZ entanglement. Further, we analyze the theory of visibility optimization, which is proven by the experimental results. The implementation of a high intensity of three-photon entangled source is a significant step towards practical long-distance multiparty quantum communication in the future.

\section{Acknowledgments}

This work was supported by the Natural Science Foundation of China (Grant No. 60878001) and by the Natural Science Foundation of Shandong Province (Grant No. Y2006A24).
[1] J.-W. Pan, Z.-B. Chen, M. Żukowski, H. Weinfurter, and A. Zeilinger, e-print arXiv:0805.2853 1 .

[2] R. Horodecki, P. Horodecki, M. Horodecki, and K. Horodecki, e-print arXiv:quant-ph/0702225 2 .

[3] E. Knill, R. Laflamme, and G.J. Milburn, Nature (London) 409, 46 (2001).

[4] P. Kok, W.J. Munro, K. Nemoto, T.C. Ralph, J.P. Dowling, and G.J. Milburn, Rev. Mod. Phys. 79, 135 (2007).

[5] C.H. Bennett, G. Brassard, C. Crépeau, R. Jozsa, A. Peres, and W.K. Wootters, Phys. Rev. Lett. 70, 1895 (1993).

[6] N. Gisin, G. Ribordy, W. Tittel, and H. Zbinden, Rev. Mod. Phys. 74, 145 (2002).

[7] D.M. Greenberger, M.A. Horne, and A. Zeilinger, in Bell's Theorem, Quantum Theory, and Conceptions of the Universe, edited by M. Kafatos (Kluwer Academic, Dordrecht, 1989), p. 69.

[8] D.M. Greenberger, M.A. Horne, A. Shimony, and A. Zeilinger, Am. J. Phys. 58, 1131 (1990).

[9] D. Bouwmeester, J.-W. Pan, M. Daniell, H. Weinfurter, and A. Zeilinger, Phys. Rev. Lett. 82, 1345 (1999); J.W. Pan, D. Bouwmeester, M. Daniell, H. Weinfurter, and A. Zeilinger, Nature (London) 403, 515 (2000).

[10] J.-W. Pan, M. Daniell, S. Gasparoni, G. Weihs, and A. Zeilinger, Phys. Rev. Lett. 86, 4435 (2001); M. Eibl,
S. Gaertner, M. Bourennane, C. Kurtsiefer, M. Żukowski, and H. Weinfurter, ibid. 90, 200403 (2003); Z. Zhao, T. Yang, Y.-A. Chen, A.-N. Zhang, M. Żukowski, and J.-W. Pan, ibid. 91, 180401 (2003).

[11] Z. Zhao, Y.-A. Chen, A.-N. Zhang, T. Yang, H.J. Briege, and J.-W. Pan, Nature (London) 430, 54 (2004).

[12] Q. Zhang, A. Goebel, C. Wagenknecht, Y.-A. Chen, B. Zhao, T. Yang, A. Mair, J. Schmiedmayer, and J.-W. Pan, Nat. Phys. 2, 678 (2006); C.-Y. Lu, X.-Q. Zhou, O. Gühne, W.-B. Gao, J. Zhang, Z.-S. Yuan, A. Goebel, T. Yang, and J.-W. Pan, ibid. 3, 91 (2007).

[13] J. G. Rarity and P. R. Tapster, Phys. Rev. A 59, R35 (1999).

[14] P.G. Kwiat, K. Mattle, H. Weinfurter, A. Zeilinger, A.V. Sergienko, and Y. Shih, Phys. Rev. Lett. 75, 4337 (1995).

[15] C.K. Hong, Z.Y. Ou, and L. Mandel, Phys. Rev. Lett. 59, 2044 (1987).

[16] N.D. Mermin, Phys. Rev. Lett. 65, 1838 (1990).

[17] A. Acín, D. Bruß, M. Lewenstein, and A. Sanpera, Phys. Rev. Lett. 87, 040401 (2001).

[18] M. Hillery, V. Bužek, and A. Berthiaume, Phys. Rev. A 59, 1829 (1999); Y.-A. Chen, A.-N. Zhang, Z. Zhao, X.Q. Zhou, C.-Y. Lu, C.-Z. Peng, T. Yang, and J.-W. Pan, Phys. Rev. Lett. 95, 200502 (2005). 